\title{
Speech Perception and Production in Cochlear Implant Recipients with Pendred Syndrome
}

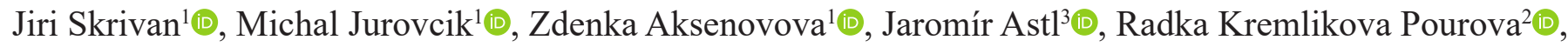 \\ Petra Dytrych ${ }^{1}$, Tomas Sieger ${ }^{4}(0)$
}

\begin{abstract}
${ }^{1}$ Department of Ear, Nose and Throat, Second Faculty of Medicine, Charles University in Prague and Motol University Hospital, Prague, Czechia ${ }^{2}$ Department of Biology and Medical Genetics, Second Faculty of Medicine, Charles University in Prague and Motol University Hospital, Prague, Czechia ${ }^{3}$ Department of Otorhinolaryngology and Maxillofacial Surgery, Third Faculty of Medicine, Charles University in Prague and Military University Hospital, Prague, Czechia

${ }^{4}$ Department of Neurology and Center of Clinical Neuroscience, First Faculty of Medicine, Charles University in Prague and Motol University Hospital, Prague, Czechia
\end{abstract}

\begin{abstract}
Aims: Patients with the Pendred syndrome suffer very often from a hearing loss. They may be good candidates for a cochlear implantation, but unfortunately, due to the fluctuating character of the hearing loss, they may escape such indication. In the study, we compared speech production and speech acquisition in 2 groups of implanted patients: those with the Pendred syndrome, and standard non-syndromic patients.

Methods: Ten patients with Pendred syndrome were analyzed for speech perception and production. The control group consisted of 41 non-syndromic implanted patients. All implantees were scored according to speech perception, speech production, and the sum of both. The data were statistically analyzed.
\end{abstract}

Results: No statistical difference was found in language acquisition and production in implantees with Pendred syndrome when compared to non-syndromic patients with cochlear implants. Nor there was any difference in speech production and acquisition between the 2 compared groups regarding surgical age, time elapsed after surgery, or age during the testing.

Conclusion: In this study evaluating language and speech production and acquisition, patients with Pendred syndrome who underwent cochlear implants show comparable results to their implanted peers with deafness of a different etiology.

\section{INTRODUCTION}

Pendred syndrome (PS) (OMIM \#274600) is an autosomal recessive hereditary disease characterized by a combination of a sensorineural hearing loss, vestibular disorders, and a non-endemic dyshormonogenetic goiter.

In PS, hearing impairment is either prelingual, severe, or profound in the majority, or postlingual and progressive. It is accompanied by temporal bone anomalies - an enlarged vestibular aqueduct (EVA), and occasionally a "Mondini deformity" (the more recent definition being an incomplete partition type II malformation, IP-II). ${ }^{1}$ The thyroid gland involvement in PS usually manifests itself in the second decade of life as a euthyroid or hypothyroid goiter. A radiological diagnosis of EVA with or without cochlear involvement does not equate to a clinical diagnosis of PS syndrome, since there are other causes of these types of temporal bone malformations without an associated thyroid disease. ${ }^{2} \mathrm{PS}$ is caused by a mutation in the SLC26A4 gene (also called the PDS gene, *OMIM 605646) which codes the anion transporter pendrin. ${ }^{3}$ This anion transporter is functional mainly in the thyroid gland and the inner ear. ${ }^{4}$ Apart from PS, mutations in SLC26A4, also cause nonsyndromic hearing loss connected with EVA (NSEVA), termed AR (autosomal recessive) hereditary non-syndromic hearing loss type 4 (DFNB4, OMIM \#600791). ${ }^{5}$ The molecular diagnosis of PDS/ NSEVA is established by the identification of biallelic pathogenic variants in SLC26A4, or a double heterozygosity for one pathogenic variant in SLC26A4 and one pathogenic variant in either FOXI1 or KNCJ10., 2,6,7

Address for Correspondence: Jiri Skrivan, Department of Ear, Nose and Throat, Second Faculty of Medicine, Charles University in Prague and Motol University Hospital e-mail: Jiri.Skrivan@fnmotol.cz

Received: November 2, 2020 Accepted: May 7, $2021 \cdot$ DOI: 10.5152/balkanmedj.2021.20182

Available at www.balkanmedicaljournal.org

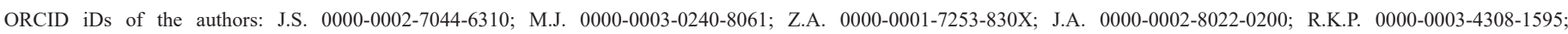
P.D. 0000-0003-2124-6766; T.S. 0000-0003-4960-1934.

Cite this article as:

Skrivan J, Jurovcik M, Aksenovova Z, et al. Speech perception and production in cochlear implant recipients with pendred syndrome. Balkan Med J. 2021;38(4):244-248.

Copyright@Author(s)-Available online at http://balkanmedicaljournal.org/ 
PS is the most common cause of syndromic hearing loss and is responsible for almost $10 \%$ of the cases of congenital deafness. ${ }^{8-10}$

In the early days of cochlear implants, patients with inner ear malformations were excluded from cochlear implant programs, since surgical risks such as a gusher were expected and audiological outcomes were considered low. ${ }^{11}$ However, later experience has shown that a cochlear implantation in children with inner ear anomalies (such as IP-II or EVA) can be surprisingly beneficial. ${ }^{12-15}$

The aim of this study was to learn whether patients with PS would represent good candidates for a cochlear implantation.

\section{MATERIAL AND METHODS}

A retrospective view of all patients with PS was performed in a tertiary referral center.

All clinical investigations were performed according to the principles of the Declaration of Helsinki. ${ }^{16}$ Since this was a retrospective study and all data were anonymised during processing, the Ethics Committee Approval and Informed Consent was not necessary.

\section{Group of Patients With PS}

Demographic data for the implanted patients, the type of deafness at the time of surgery (prelingual or postlingual), presence of temporal bone anomalies on magnetic resonance imaging (MRI)/ computed tomography (CT) scans, details of the surgery itself (including complications), and information on the outcome of implantation were retrieved from individual patient files.

The inclusion criterion was based on the presence of SLC26A4/ $P D S$ gene mutations confirmed by Sanger sequencing. ${ }^{8}$ The genetic analysis was positive in 19 patients with PS. Out of this number, 11 had been implanted, since they fulfilled the criteria for a cochlear implantation. All of these patients were operated on between 2002 and 2013.

There were 4 males and 7 females in the PS group. Seven patients were born deaf, having been classified with prelingual deafness. Their average age at surgery was 3.1 years (range 2-4 years).

Postlingually deaf patients suffered from a progressive hearing loss, and once they reached the cutoff age for the progression from severe to profound hearing loss (PTA > 80 dB HL; WHO 1991/ www.who.int/deafness/en), they had been indicated for an implant. Their average age at surgery was 9.5 years (range 5-13 years).

\section{Group of Patients Without PS}

The control group of non-PS patients was selected from implantees by random choice to be as closely matched to the PS group as possible with respect to onset of hearing impairment, gender, and age of implantation. The control group was set by a random mechanical selection from a total of 397 implanted subjects operated in the Department between the years 2000 and 2012. Patients with multiple handicaps were excluded from the selection.

The group of prelingually deaf patients consisted of 26 persons (11 males and 15 females), while the group of postlingually deaf patients consisted of 15 patients (5 males and 10 females). The average age at surgery in the prelingual group was 2 years (range $1-4$ ), and in the postlingual group was 10 years (range 3-18). One child with prelingual deafness (implanted at the age of 3 years) had partially developed speech before the surgery, and was classified as postlingual.

In the PS group, the presence of the bilateral EVA on MRI or HRCT (high resolution computer tomography) scans was diagnosed in 9 patients, there were no data about EVA in 2 patients. In the control group, there were no cases of inner ear anomalies. Regarding intraoperative and early postoperative complications in the PS group, there was one case of a bilateral gusher, which was solved by tight muscle packing around an electrode in the round window. In the control group, there were no intraoperative complications; nevertheless, there were 2 cases of a wound hematoma which occurred early after the surgery. It was solved by a wound puncture and compression.

Rehabilitation of all implanted patients was undertaken both before and after surgeries in our cochlear implantation center.

\section{Evaluation of Speech and Hearing}

To evaluate the general speech perception and production, we developed our own scoring system, called the Speech Perception and Production Score, Motol Speech Perception and Production Score (MSPPS). The test evaluates both speech perception and production, with a score of zero representing no speech and perception, and a score of 5 representing the speech and perception of a person with normal hearing. Adding both the values together shows the global speech and hearing status.

The scoring system was evaluated for validity and reliability. During testing, all implantees displayed language development comparable to their peers. Therefore, the MSPPS test represented a suitable method for the measurement of outcomes.

\section{Statistical Analysis}

The Wilcoxon exact rank-sum test (Streitberg-Röhmel "shift" algorithm) was used for intergroup comparison of age at surgery and the time elapsed between the surgery and the evaluation. Fisher's exact test was used to compare sex distribution between groups. To compare speech between the PS and non-PS patient groups, we successively analyzed the ordinal outcomes of speech acquisition and speech understanding, and the sum of those 2 scores, using proportional odds logistic regression. The patient group and the status of prelingual/postlingual deafness were considered to be covariates. The likelihood ratio test was used to assess the significance of the covariates of interest. The level of significance was set to 5\%. Statistical analysis was carried out in R [R Core Team (2021). R: A language and environment for statistical computing. R Foundation for Statistical Computing, Vienna, Austria. URL https://www.R-project.org/].

We performed a simple post hoc power analysis for power estimation and sample size estimation. In both cases, we generated bootstrap samples for both groups by sampling with replacement from the observed samples. For sample size estimation, we repeatedly generated bootstrap samples of increasing size for both groups, and compared speech perception between the generated groups 
of hypothetical patients. The desired sample size was estimated as the sample size reaching a significant group difference in $80 \%$ of repetitions.

\section{RESULTS}

Two groups of implanted patients, PS and nonPS, were compared in terms of their speech perception and production. Each group was divided into prelingually and postlingually deaf.

The Pendred group consisted of 11 implantees, 7 prelingually deaf and 4 postlingually deaf. One patient from this group was not evaluated, as she was implanted in another center and was lost in follow-up. The non-PS group consisted of 41 patients, 26 prelingually deaf and 15 postlingually deaf.

The mean age at surgery was 5.3 years (median 4 years, range 2-13 years) in the PS group, and 5.1 years (median 3 years, range $1-18$ years $)$ in the non-PS group $(P=.36)$. The groups did not differ in terms of sex $(P=1)$. The mean time elapsed between surgery and evaluation was 13.2 years (median 13 years, range 7-18 years) in the PS group, and 13.3 years (median 13 years, range $8-20$ years $)$ in the non-PS group $(P=.52)$. From this point of view, a longer time elapsed after surgery ensured a stable outcome.

We found no difference in speech between the PS $(n=10)$ and nonPS $(n=41)$ groups.

Speech understanding did not differ between the groups (PS vs. non-PS odds ratio $\left.0.5,95 \% \mathrm{CI}(0.1,1.9), \chi^{2}(1)=1.055, P=.30\right)$. The speech understanding scores were only insignificantly higher in the PS group (minimal score 3, mean 4.3, and median 5) compared to the non-PS patients (minimal score 2, mean 4.0, and median 4). Speech production also did not differ between the groups (PS vs. non-PS odds ratio $\left.1.1,95 \% \mathrm{CI}(0.3,4.1), \chi^{2}(1)=0.045, P=.83\right)$. Likewise, the sum of the speech understanding and production scores did not differ between the groups $\left(\chi^{2}(1)=0.118, P=.73\right)$. The prelingual/postlingual deafness status did not affect any of the speech scores.

The distribution of MSPPS scores for the Pendred and non-Pendred groups, prelinguals, and postlinguals, are shown in Figures 1-3.

The post hoc power analysis based on speech perception estimated the power of this study to be $26 \%$, with an effect size of Cohen's $d$ of 0.35 . The post hoc power analysis for sample size estimation based on speech perception revealed the necessary sample size to be at least 100 PS and 200 non-PS patients to reach a power of $80 \%$. However, a much larger sample size would be needed if the hypothetical larger sample does not resemble the observed sample of 10 PS +41 non-PS patients.

\section{DISCUSSION}

The aim of this paper was to compare speech perception and speech production between 2 groups of implanted patientspatients with the PS and patients with standard non-syndromic hearing loss.

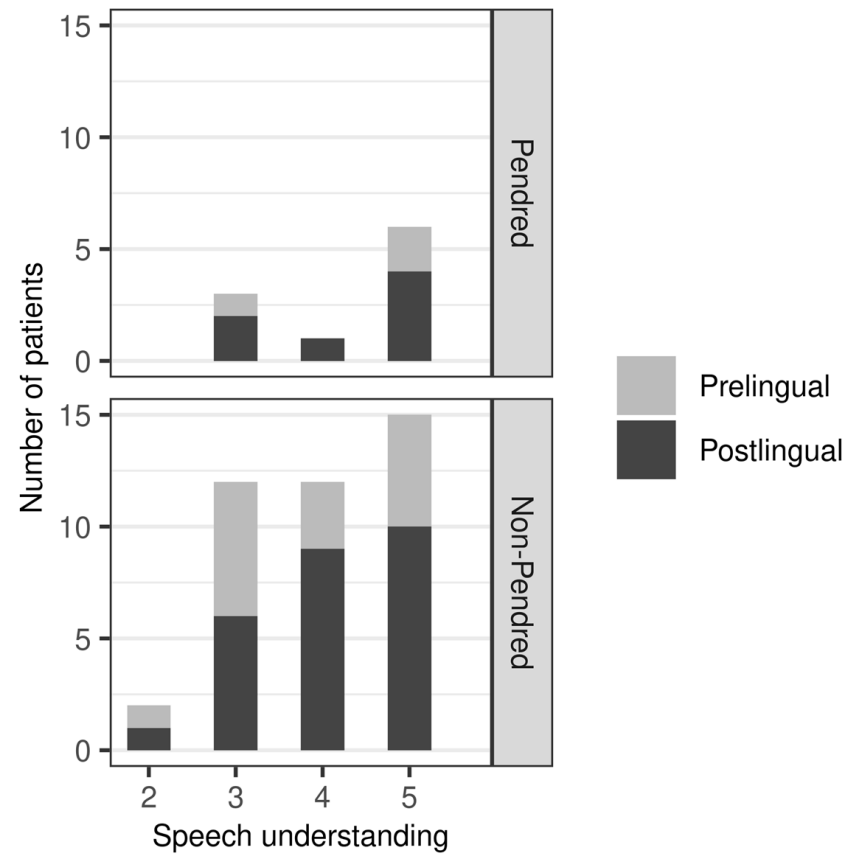

FIG. 1. Distribution of speech understanding scores in MSPPS for Pendred and non-Pendred groups, both prelinguals and postlinguals. The higher the number, the better the outcome.

In prelinguals, the implant age in the PS group was insignificantly higher than the implant age in the non-PS group. Prelingually deaf children are usually detected by a neonatal hearing screening and are quickly directed toward cochlear implantation or hearing aids.

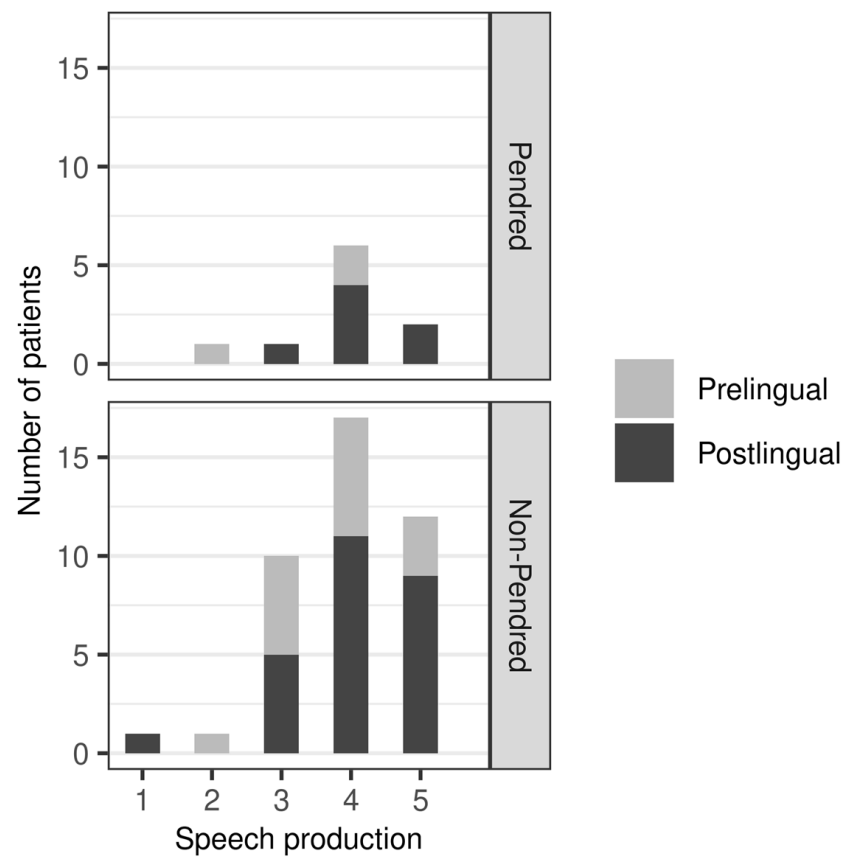

FIG. 2. Distribution of speech production scores in MSPPS for Pendred and non-Pendred groups, both prelinguals and postlinguals. The higher the number, the better the outcome. 


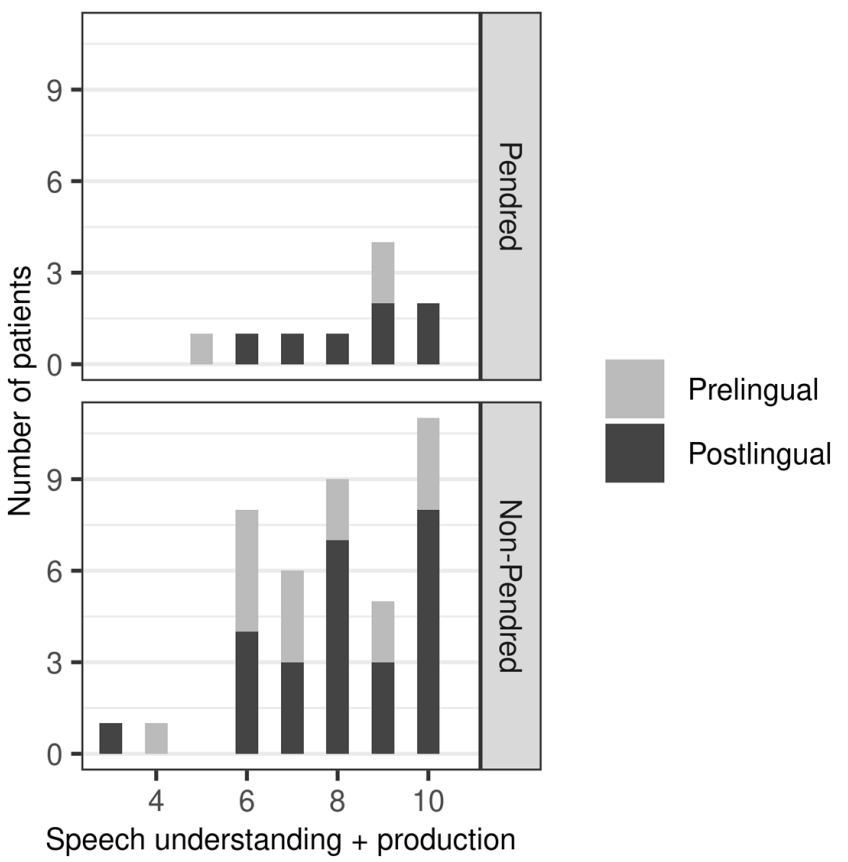

FIG. 3. Distribution of total scores in MSPPS (sum of speech production and perception scores) for Pendred and non-Pendred groups, both prelinguals and postlinguals. The higher the number, the better the outcome.

Such children are usually implanted at the age of 12-13 months. On the other hand, it may be generally said that $50 \%$ of PS individuals who were screened after birth may pass a hearing test at least on one side. ${ }^{17}$ They suffer from a progressive or fluctuating hearing loss and may end up with hearing aids, and a decision to implant may be delayed. A well-known fact is that an early implantation in prelingually deaf children definitely yields better outcomes. As a rule of thumb, the same applies to the interval between the onset of deafness and cochlear implantation in postlingually deaf individuals. The fluctuating character of hearing loss in PS patients may prolong time in counseling, between candidacy consideration and implantation. Children who are diagnosed with NSEVA by imaging (MRI, CT), should be quickly analyzed for PS.

In the present study, no statistical difference was shown in speech production and perception between the PS and the non-PS group. Nevertheless, the analysis may be flawed by the low numbers of patients. Modeling the situation on a large cohort brought the same results. Moreover, some of the tested subjects had a mixed congenital pathology, which may have contributed to the groups being non-cohesive.

Few studies evaluating cochlear implantation outcome in PS patients have been published. The designs of these studies are different, with comparison of PS versus NSEVA groups, ${ }^{18}$ comparison of children with isolated EVA versus EVA and other bony abnormalities, ${ }^{13}$ or just general remarks on specific (including surgical) problems in cochlear implantation in children with PS. ${ }^{19}$ PS and cochlear implantation have been studied by a Danish group..$^{14,17}$ Differing from our study, they reported that children with PS perform below age-equivalent measures for receptive vocabulary outcome when compared to both children with normal hearing and children with non-syndromic mixed hereditary congenital hearing impairment who receive cochlear implants earlier. ${ }^{20}$

In children with PS, the following points should be considered as being of the utmost importance:

- Early detection of the pathology by genetic testing.

- Temporal bone morphology by CT and MRI.

- Early implantation in prelinguals.

- Focus on thyroid gland morphology and function.

No statistical difference was found in implanted children with PS when compared to standard implantees with a different etiology of deafness. Children with PS deserve special observation regarding the proper timing for a cochlear implant and temporal bone pathology, and last but not least, a life-long follow-up of hearing status and thyroid gland pathology.

Ethics Committee Approval: Ethics committee approval was received for this study from the Ethics Committee of the University Hospital Motol and 2nd School of Medicine, Charles University in Prague (EK-791/21).

Patient Consent for Publication: Informed consent have been sent to all the participants and only those approved had been included in the study.

Author Contributions: Concept - J.S., M.A., R.K.P.; Design - J.S., M.A.; Supervision J.S., M.A., Z.A.; Resources - J.S., M.J.; Materials - Z.A.; Data Collection and/or Processing - T.S., Z.A.; Analysis and/or Interpretation - J.S., T.S., R.K.P.; Literature Review - J.S., M.J., R.K.P.; Writing - J.S., T.S.; Critical Review - J.S., M.J., T.S.

Data-sharing Statement: The data that support the findings of this study are available on request from the corresponding author. The data are not publicly available due to privacy or ethical restrictions.

Conflict of Interest: The authors declare that they have no conflicts of interest concerning this article.

Funding: This paper is supported by the Ministry of Health, Czech Republic - Conceptual Development of Research Organization, Motol University Hospital, Prague, grant 00064203 and by the Ministry of Health, Czech Republic (Grant NU21-01-00448).

\section{REFERENCES}

1. Sennaroglu L, Saatci I. A new classification for cochleovestibular malformations Laryngoscope. 2002;112(12):2230-2241 . [CrossRef]

2. Smith RJH. Pendred syndrome/nonsyndromic enlarged vestibular aqueduct. In: Adam MP et al., eds. GeneReviews ${ }^{\circledR}$. Seattle (WA): University of Washington; 1993.

3. Everett LA, Glaser B, Beck JC, et al. Pendred syndrome is caused by mutations in a putative sulphate transporter gene (PDS). Nat Genet. 1997;17(4):411-422. [CrossRef]

4. Scott DA, Wang R, Kreman TM, et al. Functional differences of the PDS gene product are associated with phenotypic variation in patients with Pendred syndrome and non-syndromic hearing loss (DFNB4). Hum Mol Genet. 2000;9(11):1709-1715. [CrossRef]

5. Li XC, Everett LA, Lalwani AK, et al. A mutation in PDS causes non-syndromic recessive deafness. Nat Genet. 1998;18(3):215-217. [CrossRef]

6. Yang T, Gurrola JG, Wu H, et al. Mutations of KCNJ10 together with mutations of SLC26A4 cause digenic nonsyndromic hearing loss associated with enlarged vestibular aqueduct syndrome. Am J Hum Genet. 2009;84(5):651-657. [CrossRef]

7. Yang T, Vidarsson H, Rodrigo-Blomqvist S, et al. Transcriptional control of SLC26A4 is involved in Pendred syndrome and nonsyndromic enlargement of vestibular aqueduct (DFNB4). Am J Hum Genet. 2007;80(6):1055-1063. [CrossRef] 
8. Pourová R, Janousek P, Jurovcík M, et al. Spectrum and frequency of SLC26A4 mutations among Czech patients with early hearing loss with and without enlarged vestibular aqueduct (EVA). Ann Hum Genet. 2010;74(ych4):299-307. [CrossRef]

9. Katra R, Pourova RK, Dytrych P, et al. Pendredův syndrome v České republice Pendred syndrome in the Czech Republic. Otorinolaringol Foniatrie, 2011;60(2):103111.

10. Astl J, Stolbova D, Skrivan J, Holcat M. Struma spojená s nedoslýchavostí - Goitre associated with deafness. Otorinolaringol Foniatrie. 1996;45(1):31-34.

11. Buchman CA, Copeland BJ, Yu KK, et al. Cochlear implantation in children with congenital inner ear malformations. Laryngoscope. 2004;114(2):309-316. [CrossRef]

12. Sennaroglu L, Sarac S, Ergin T. Surgical results of cochlear implantation in malformed cochlea. Otol Neurotol. 2006;27(5):615-623. [CrossRef]

13. Pritchett C, Zwolan T, Huq F, et al. Variations in the cochlear implant experience in children with enlarged vestibular aqueduct. Laryngoscope. 2015;125(9):2169-2174. [CrossRef]

14. Mey K, Bille M, Cayé-Thomasen P. Cochlear implantation in Pendred syndrome and non-syndromic enlarged vestibular aqueduct - clinical challenges, surgical results, and complications. Acta Otolaryngol. 2016;136(10):1064-1068. [CrossRef]
15. Catli T, Uckan B, Olgun L. Speech and Language development after cochlear implantation in children with bony labyrinth malformations: long-term results. Eur Arch Otorhinolaryngol. 2015;272(11):3131-3136. [CrossRef]

16. WMA Declaration of Helsinki - ethical principles for medical research involving human subjects. JAMA. 2013;310(20):2191-2194. Available at: https://www.wma .net/policies-post/wma-declaration-of-helsinki-ethical-principles-for-medical-researc h-involving-human-subjects/. [CrossRef]

17. Mey K, Bille M, Rye Rasmussen SH, Tranebjærg L, Cayé-Thomasen P. The Natural History of Hearing Loss in Pendred Syndrome and Non-Syndromic Enlarged Vestibular Aqueduct. Otol Neurotol. 2019;40(3):e178-e185. [CrossRef]

18. van Nierop JWI, Huinck WJ, Pennings RJE, et al. Patients with Pendred syndrome: is cochlear implantation beneficial? Clin Otolaryngol. 2016;41(4):386-394. [CrossRef]

19. Kontorinis G, Lenarz T, Lesinski-Schiedat A, Neuburger J. Cochlear implantation in Pendred syndrome. Cochlear Implants Int. 2011;12(3):157-163. [CrossRef]

20. Mey K, Percy-Smith L, Hallstrøm M, Sandvej M, Cayé-Thomasen P. Receptive language acquisition in a pediatric population with Pendred syndrome and non-syndromic enlarged vestibular aqueduct. Acta Otolaryngol. 2020;140(1):46-50. [CrossRef] 